Bundesgesundheitsbl $2021 \cdot 64: 277-284$ https://doi.org/10.1007/s00103-021-03282-4 Eingegangen: 10 . September 2020

Angenommen: 14. Januar 2021

Online publiziert: 12. Februar 2021

c c Der/die Autor(en) 2021

Eva Maria Bitzer' $\cdot$ Lena Ansmann ${ }^{2} \cdot$ Madlen Hörold $^{3} \cdot$ Lisa Lyssenko' $^{1}$ Christian Apfelbacher ${ }^{3}$

'Institut für Alltagskultur und Bewegung und Gesundheit, Fachrichtung: Public Health \& Health Education, Pädagogische Hochschule Freiburg, Freiburg, Deutschland

${ }^{2}$ Fakultät VI Medizin und Gesundheitswissenschaften, Organisationsbezogene Versorgungsforschung, Carl von Ossietzky Universität Oldenburg, Oldenburg, Deutschland

${ }^{3}$ Medizinische Fakultät, Institut für Sozialmedizin und Gesundheitssystemforschung, Otto-von-GuerickeUniversität Magdeburg, Magdeburg, Deutschland

\title{
„... oder doch lieber daheimbleiben?" - Unterstützung bei der Entscheidung zur Inanspruchnahme der Regelversorgung während der COVID-19-Pandemie durch Akteure des Gesundheitssystems
}

sondern auch weniger in Anspruch genommen wird [14].

Bei der (Nicht-)Inanspruchnahme von Gesundheitsleistungen in der Pandemie spielen Ängste und Ungewissheit eine zentrale Rolle. Aktuelle Studien berichten u.a. von der Intention, das Gesundheitssystem zu entlasten [15], Angst vor erhöhtem Infektionsrisiko [16], Unsicherheit und fehlendem Wissen, wie die Informationen der Bundesregierung und aus anderen Quellen einzuschätzen sind [15], und der Sorge, bei einem stationären Aufenthalt aufgrund der Besuchsverbote unter Isolation und Einsamkeit $\mathrm{zu}$ leiden [17]. Daten aus der SARSPandemie 2002/2003 legen nahe, dass die (Nicht-)Inanspruchnahme mit dem Fallgeschehen oszilliert, jedoch keine realistische Risikowahrnehmung abbildet [18]. In der Anfangszeit der COVID-19Pandemie war das nosokomiale Infektionsrisiko laut einer Metaanalyse hoch - 4 von 10 stationär behandelte SARS-CoV2-Infektionen waren auf eine Infektion im Krankenhaus zurückführen [19,
20]. Ob die Nichtinanspruchnahme von Gesundheitsleistungen in bestimmten Bereichen sachgerecht ist und/oder $\mathrm{zu}$ einem Abbau von Überversorgung führt [21], ist für den Einzelfall wenig relevant. Für die adäquate Inanspruchnahme in der Pandemie ist die individuelle $\mathrm{Ge}$ sundheitskompetenz entscheidend, d.h. die Fähigkeit, gesundheitliche Information $\mathrm{zu}$ suchen, $\mathrm{zu}$ finden, zu verstehen, $\mathrm{zu}$ bewerten und für gesundheitsbezogene Entscheidungen zu nutzen [22]. Bei den aktuell tiefgreifenden Auswirkungen der COVID-19-Pandemie auf das gesellschaftliche, wirtschaftliche und soziale Leben stellt insbesondere die kritische Gesundheitskompetenz, also die Fähigkeit, Gesundheitsinformationen abzuwägen und in den eigenen Kontext $\mathrm{zu}$ überführen, eine wichtige Ressource dar [23, 24]. Bereits ohne Pandemie ist Gesundheitskompetenz in der Gesellschaft ungleich verteilt, so verfügen sozioökonomisch benachteiligte Bevölkerungsgruppen häufig über eine geringere Gesundheitskompetenz 
[25-28]. In der Pandemie verschärfen sich die Lebensbedingungen noch einmal, so sind benachteiligte Bevölkerungsgruppen überproportional von der Pandemie und den pandemiebedingten Einschränkungen betroffen. Es wird damit für sie umso schwerer, angemessene Entscheidungen über die Inanspruchnahme zu treffen, sowohl im direkten Zusammenhang mit COVID19, aber auch bei nichtübertragbaren Erkrankungen in der Regelversorgung.

Die organisationale Gesundheitskompetenz (oGK) bezieht sich auf den Grad, zu dem das Gesundheitssystem bzw. seine Einrichtungen - beispielsweise durch aktive Gestaltung von Strukturen und Prozessen gesundheitlicher Versorgung - Bedingungen schaffen, in denen Subsysteme und/oder Individuen angemessene gesundheitsbezogene Entscheidungen treffen bzw. ihren eigenen Kontext gesundheitsförderlich gestalten können [29, 30]. oGK meint nicht, dass Versorgungsorganisationen selbst "gesundheitskompetent" agieren, sondern dass sie ihren Patient*innen Gesundheitskompetenz ermöglichen [31]. Mit dieser Ausrichtung weist oGK Bezüge zu Konzepten wie Responsivität gesundheitlicher Versorgung [32] und allgemein der Patient*innenorientierung auf [33] und, wenn es um die Umsetzung geht, zu Managementsystemen [34].

Die Pandemie fordert die oGK der Gesundheitssysteme auf der Makro-, Meso- und Mikroebene der gesundheitlichen Versorgung. Dabei sind alle Ebenen selbst von der Pandemie betroffen und es gilt auf allen Ebenen abzuwägen zwischen Gewährleistung der Versorgung von COVID-19 und der Aufrechterhaltung der Regelversorgung bei Minimierung des Infektionsrisikos.

Makroebene. Pandemiemanagement auf der Makroebene - in der Bevölkerung und innerhalb des Gesundheitsversorgungssystems - liegt in der Verantwortung von Politik auf Landes- und Bundesebene sowie der Gremien der Selbstverwaltung. Ist es erfolgreich, reduziert es die Wahrscheinlichkeit kritischer Situationen in der kurativen Versorgung (Überlastung, nosokomiale Infektionen) auf ein Minimum und senkt die Ein- schränkungen in der Versorgung, sodass weniger Menschen Abwägungsprozesse in Hinblick auf die Inanspruchnahme treffen müssen, die für sie u. U. schwierig sind. Erfolgreiches Pandemiemanagement erleichtert damit gesundheitskompetentes Agieren unabhängig von der individuellen Gesundheitskompetenz [35]. In Deutschland waren die Einschränkungen des Versorgungsangebots (bislang) zeitlich eng begrenzt und die Notfallversorgung durchgehend sichergestellt.

Mesoebene. Auf der Angebotsseite sind Gesundheitsleistungen in mehrfacher Hinsicht von der Pandemie geprägt. Auch bei niedrigem Infektionsgeschehen sind Anbieter und professionell Tätige mit der Herausforderung konfrontiert, bislang wenig bekannte - teils wechselnde - Vorgaben zum Infektionsschutz in Einrichtungen umzusetzen und ggf. gegenüber Patient*innen und Angehörigen zu vertreten. Ihnen kommt eine zentrale Rolle zu, weil sie den konkreten Kontext gestalten, in dem Versorgung stattfindet. Bei der Förderung von oGK auf der Mesoebene werden Maßnahmen adressiert, die geeignet sind, das Infektionsrisiko in Einrichtungen der gesundheitlichen Versorgung niedrig zu halten, z.B. im Operationsaal [36], bei Vorkehrungen zur Infektionskontrolle in der Krankenversorgung [37] und bei telemedizinischen Interventionen [38]. Effektiv umgesetzt reduzieren sie das Risiko nosokomialer Infektionen und erleichtern so den Nicht-an-COVID19-Erkrankten die Entscheidung zur Inanspruchnahme.

Mikroebene. Die Förderung der oGK manifestiert sich in den Bemühungen der Einrichtungen der gesundheitlichen Versorgung bzw. des Gesundheitssystems, der Komplexität der Bedürfnisse, Fähigkeiten, Präferenzen und medizinischen Bedarfe von Patient ${ }^{*}$ innen gerecht zu werden [39] und diesen in einer von Unsicherheit geprägten Pandemiesituation bestmöglich gesundheitskompetente Entscheidungen zu ermöglichen [39]. Auf der Mikroebene zeigt sich die Förderung der oGK u. a. darin, ob Einrichtungen ihre Zielgruppe zu dem Thema informieren, ob diese Informationen ein- fach gefunden und leicht $\mathrm{zu}$ verstehen sind und ob sie die für die Abwägung im Entscheidungsfindungsprozess relevanten Informationen beinhalten und $\mathrm{zu}$ welchem Grad sie die Kriterien für gute Patient*inneninformationen [40] erfüllen.

\section{Ziel/Fragestellung}

Zielsetzung des vorliegenden Beitrags ist eine Bestandsaufnahme zur Unterstützung der oGK in der Regelversorgung nichtübertragbarer Erkrankungen in der ersten Welle der COVID-19-Pandemie: Inwiefern wurden Menschen mit gesundheitlichen Beschwerden dabei unterstützt, gesundheitskompetente Entscheidungen für oder gegen die Inanspruchnahme von Versorgungsleistungen $\mathrm{zu}$ treffen? Der Fokus dieser Arbeit liegt dabei auf Informationen und Maßnahmen, die zum einen die betroffenen Patient*innen selbst adressieren (Mikroebene). Zum anderen wird auch betrachtet, inwiefern Informationen und Maßnahmen Einrichtungen der gesundheitlichen Versorgung adressieren (Mesoebene), um so indirekt Patient ${ }^{*}$ innen dabei $\mathrm{zu}$ unterstützen, gesundheitskompetente Entscheidungen zu treffen.

Erste Überlegungen zu dieser Frage haben wir in einem Hintergrundpapier für das Kompetenznetz Public Health zu COVID-19 dargelegt [41]. Für den vorliegenden Beitrag haben wir die Argumentation geschärft, zusätzliche Informationen herangezogen und die Ergebnisse aktualisiert.

\section{Methoden}

Operationalisiert haben wir oGK über die Informationen, die ausgewählte Akteure für Adressaten auf Meso- und Mikroebene zur Gesundheitsversorgung von Menschen ohne (Verdacht auf) eine COVID-19-Erkrankung im Internet zur Verfügung stellen. Die Stichprobe wurde nach fachlicher und berufsrechtlicher Zuständigkeit von Institutionen/ Organisationen gebildet. Die Datenauswertung erfolgte über ein inhaltsanalytisches Rating in theoriegeleiteten Antwortkategorien. 
Bundesgesundheitsbl 2021 ·64:277-284 https://doi.org/10.1007/s00103-021-03282-4

(c) Der/die Autor(en) 2021

\section{E. M. Bitzer · L. Ansmann · M. Hörold · L. Lyssenko · C. Apfelbacher}

\section{„... oder doch lieber daheimbleiben?" - Unterstützung bei der Entscheidung zur Inanspruchnahme der Regelversorgung während der COVID-19-Pandemie durch Akteure des Gesundheitssystems}

\section{Zusammenfassung}

Hintergrund. In der COVID-19-Pandemie ging die Versorgung nichtübertragbarer Erkrankungen zeitweise deutlich zurück, auch weil Menschen Angst vor einer Ansteckung hatten. Wir führen eine Bestandsaufnahme zur organisationalen Gesundheitskompetenz in der Regelversorgung nichtübertragbarer Erkrankungen in der ersten Welle der COVID-19-Pandemie durch und fragen: Inwiefern wurden Menschen mit gesundheitlichen Beschwerden dabei unterstützt, gesundheitskompetente Entscheidungen für oder gegen die Inanspruchnahme von Versorgungsleistungen zu treffen?

Methodik. Strukturierende Inhaltsanalyse der Internetpräsenzen der Mitglieder der Arbeitsgemeinschaft der Wissenschaftlich
Medizinischen Fachgesellschaften (AWMF; $n=179$ ), der kassen(zahn)ärztlichen Vereinigungen (K(Z)Ven; $n=38)$, ausgewählter Krankenkassen $(n=21)$, ausgewählter Behandlungseinrichtungen $(n=25)$ und überregionaler Anbieter von Gesundheitsinformationen $(n=5)$ zu Informationen und Angeboten zum Thema.

Ergebnisse. Die geprüften Internetpräsenzen informieren weitgehend rund um COVID19, aber nur selten darüber, wie man sich bei einer (vermuteten) anderen Erkrankung in Bezug auf die Inanspruchnahme von Versorgungsleistungen verhält. 2 Portale von Anbietern von Gesundheitsinformationen, eine Krankenkasse, aber keine der KVen bieten explizite Entscheidungshilfen an. KVen weisen öfter, aber nicht durchgängig auf die generelle Möglichkeit von Videosprechstunden hin. Diskussion. Für die meisten Patient*innen gab es damit keine gezielten Informationen zu dem Thema. Angesichts der Fortdauer der COVID-19-Pandemie gilt es, vorhandene vertrauenswürdige, qualitativ hochwertige Informations- und Beratungskapazitäten auszubauen und ihre Bekanntheit zu erhöhen, um gesundheitskompetente Entscheidungen auch in der Pandemie zu ermöglichen.

\section{Schlüsselwörter}

Individuelle Gesundheitskompetenz . Organisationale Gesundheitskompetenz . Gesundheitsinformation - Entscheidungsunterstützung · Gesundheitssystem

\section{"I better stay at home..."-health system decisions to support the use of routine healthcare during the COVID-19 pandemic}

\section{Abstract}

Background. During the COVID-19 pandemic, sharp disruptions of healthcare utilization for noncommunicable diseases were observed to some extent because of people's fear of infection. We undertook a review of German healthcare institutions asking: To what extent were people supported in their decisions (not) to seek medical care for health problems other than COVID-19?

Methods. Content analysis of the websites of the members of the Association of Scientific Medical Societies (AWMF; $n=179$ ), the association of statutory (dental) health insurance physicians (K(Z)Vs; $n=38)$, selected health insurances $(n=21)$, selected healthcare institutions $(n=25)$, and supraregional health information providers $(n=5)$ for information and offers that address people with noncommunicable diseases.

Results. The examined websites provide information about COVID-19, but only rarely about how to behave in the case of another (suspected) disease regarding healthcare utilization. Two health information provider portals, one health insurance company, but none of the KVs offer explicit decision support. KVs refer more often, but not consistently, to the general possibility of video consulting.

Discussion. Information concerning the topic is scarce for most of the patients. In the face of the ongoing pandemic, it is important to expand existing, trustworthy, high-quality information and advisory capacities to increase their profile in order to enable healthliterate decisions even during a pandemic.

\section{Keywords}

Health literacy · Health-literate healthcare Health information - Decision support Healthcare system

\section{Kategorienbildung}

Der Mesoebene wurden Informationen zugeordnet, die sich an Leistungserbringer, Fachkräfte im Gesundheitswesen, Leistungsträger und/oder Akteure in der (Gesundheits-)Politik richten. In die Teilstichprobe der Fachgesellschaften wurden Stellungnahmen und Pressemitteilungen einbezogen, wenn diese direkt von der jeweiligen Institution/ Organisation herausgegeben wurden.

Die Mikroebene wurde über Informationen abgebildet, die sich direkt an die Allgemeinbevölkerung richten und gezielt die Inanspruchnahme von Gesundheitsleistungen ohne (Verdacht auf) eine Infektion mit COVID-19 thematisieren (z. B. Ablauf von Untersuchungen, Besuchsregelungen, Kontakt zu anderen Patient*innen).

Auf beiden Ebenen wurden folgende Inhalte codiert:

- organisatorische Hinweise: Abläufe und Strukturen im Pandemiemanagement,
- Appell: Aufrufe, trotz der Pandemie medizinische Versorgung wahrzunehmen,

- Bitte: Ersuchen, nicht dringliche Behandlungsanlässe und Inanspruchnahme zu verschieben,

- allgemeine Entscheidungsunterstützung: Informationen für $\mathrm{Pa}$ tient*innen, wie Argumente für und gegen die Inanspruchnahme (bestimmter) Versorgungsleistungen gewichtet, gegenübergestellt werden,

- individuelle Beratung: Möglichkeit einer individuellen Beratung, ohne 
Tab. 1 Informationen zum Thema „Versorgung für Nicht-an-COVID-19-Erkrankte" auf Internetpräsenzen von Akteuren des Gesundheitssystems

\begin{tabular}{|c|c|c|c|c|c|c|c|c|c|c|}
\hline \multirow[t]{2}{*}{ Akteur } & \multirow{2}{*}{$\begin{array}{l}\text { Anzahl } \\
(n)\end{array}$} & \multicolumn{2}{|c|}{ Thema enthalten } & \multicolumn{2}{|c|}{ Falls Ja: adressiert an } & \multicolumn{5}{|c|}{ Inhalte } \\
\hline & & Nein & Ja & $\begin{array}{l}\text { Fachöffentlich- } \\
\text { keit/Politik } \\
\text { (Mesoebene) }\end{array}$ & $\begin{array}{l}\text { Öffentlichkeit/ } \\
\text { Patient*innen } \\
\text { (Mikroebene) }\end{array}$ & Orga. & Appell & Bitte & $\begin{array}{l}\text { Entschei- } \\
\text { dungsun- } \\
\text { terstützung }\end{array}$ & $\begin{array}{l}\text { Individuelle } \\
\text { Beratung }\end{array}$ \\
\hline Fachgesellschaften $^{a}$ & 179 & 156 & 23 & 13 & 12 & $7^{b}$ & $11^{c}$ & $4^{d}$ & 1 & 1 \\
\hline $\begin{array}{l}\text { Kassenärztliche Bundes- } \\
\text { vereinigung }\end{array}$ & 1 & - & 1 & 1 & 0 & 1 & 0 & 0 & 0 & 0 \\
\hline $\begin{array}{l}\text { Kassenärztliche Vereini- } \\
\text { gungen }\end{array}$ & 17 & 9 & 8 & 8 & 0 & $8^{e}$ & 0 & 0 & 0 & 4 \\
\hline $\begin{array}{l}\text { Kassenzahnärztliche } \\
\text { Bundesvereinigung }\end{array}$ & 1 & - & 1 & 1 & 0 & 1 & 1 & 0 & 0 & 0 \\
\hline $\begin{array}{l}\text { Kassenzahnärztliche } \\
\text { Vereinigungen }\end{array}$ & 17 & 10 & 7 & 7 & 7 & 5 & 7 & 0 & 1 & 4 \\
\hline $\begin{array}{l}\text { Gesetzliche } \\
\text { Krankenkassen }^{f}\end{array}$ & 21 & 20 & 1 & n. a. & 1 & 0 & 0 & 0 & 1 & 21 \\
\hline $\begin{array}{l}\text { Nichtkommerzielle } \\
\text { Anbieter von Gesund- } \\
\text { heitsinformationen }\end{array}$ & 5 & 2 & 3 & n. a. & 3 & n. a. & 0 & 0 & 3 & 2 \\
\hline $\begin{array}{l}\text { Krankenhäuser und } \\
\text { Rehabilitations- } \\
\text { einrichtungen }{ }^{9}\end{array}$ & 25 & 3 & 22 & n. a. & 22 & 22 & 0 & 0 & 0 & 6 \\
\hline \multicolumn{11}{|c|}{ 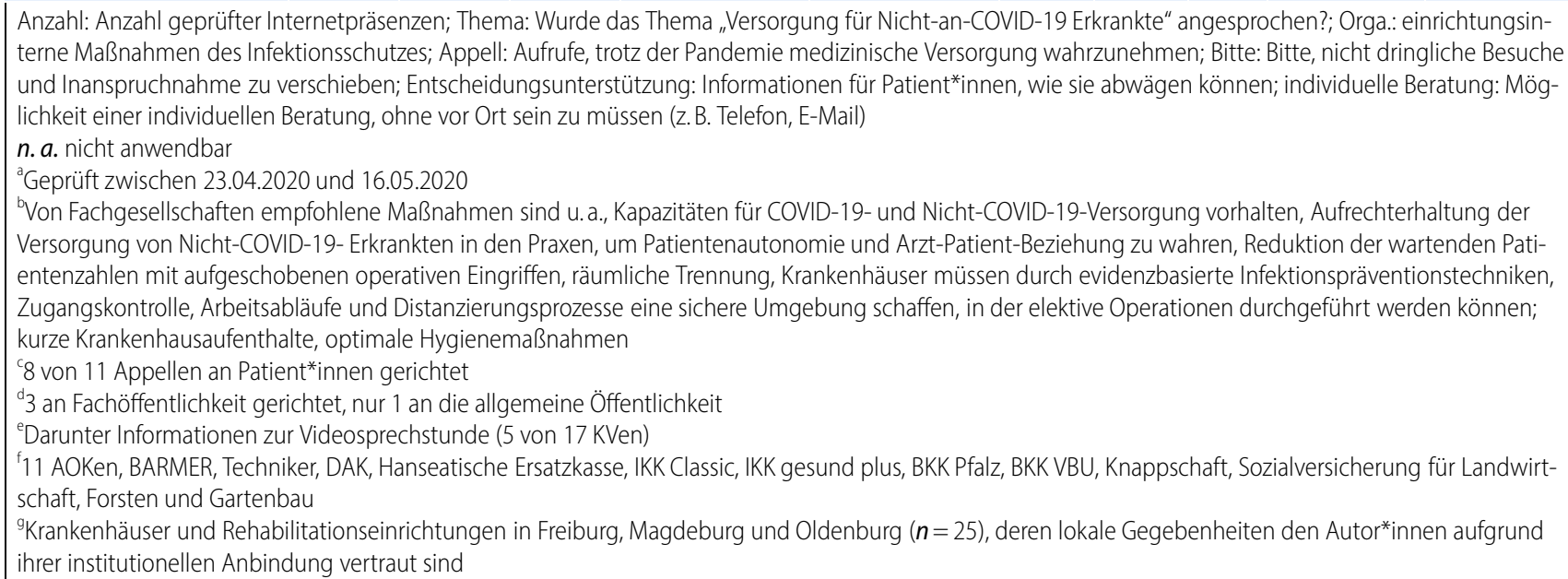 } \\
\hline
\end{tabular}

vor Ort sein zu müssen (z. B. Telefon, E-Mail).

\section{Stichprobe}

In der ersten Erhebungsphase 23.04.2020-16.05.2020 lag der Fokus auf Wissens- und Verantwortungsträgern als Akteure, die auf Makroebene wissenschaftlich fundierte Gesundheitsversorgung mitgestalten oder vertreten. In die Untersuchung eingeschlossen wurden alle Mitglieder der Arbeitsgemeinschaft der Wissenschaftlichen Medizinischen Fachgesellschaften (AWMF; $n=179)$ und kassen(zahn)ärztlichen (Bundes-)Vereinigungen $(n=36)$ sowie die größten nichtkommerziellen
Institutionen mit gesellschaftlichem/ politischem Auftrag zur Vermittlung von Gesundheitsinformationen $(n=5)$ : Institut für Wirtschaftlichkeit und Qualität im Gesundheitswesen (IQWIG), Bundeszentrale für gesundheitliche Aufklärung (BZgA), Bundesministerium für Gesundheit (BMG), Unabhängige Patientenberatung nach $\$ 65$ b SGB V (UPD), Krebsinformationsdienst am Deutschen Krebsforschungszentrum (KID).

In der zweiten Erhebungsphase vom 05.08.2020 bis 01.09.2020 wurde die Stichprobe um Kostenträger und Einrichtungen der Gesundheitsversorgung ergänzt. Eingeschlossen wurden bundesweit tätige gesetzliche Krankenkassen, deren Mitgliederzahlen eine hohe
Reichweite der Internetpräsenz nahelegen (AOK, BARMER; Techniker, DAK, Hanseatische Ersatzkasse, IKK Classic, IKK gesund plus, BKK Pfalz, BKK VBU, Knappschaft, Sozialversicherung für Landwirtschaft, Forsten und Gartenbau), sowie die Internetpräsenzen aller Krankenhäuser und Rehabilitationseinrichtungen in 3 mittelgroßen Städten (Freiburg, Magdeburg und Oldenburg; $n=25)$, deren lokale Gegebenheiten den Autor*innen aufgrund ihrer institutionellen Anbindung vertraut sind.

\section{Datenextraktion und Auswertung}

Die Datenerhebung erfolgte über die Internetpräsenzen der Institutionen/ 
Organisationen. Die Webseiten wurden von jeweils 2 Autor $^{*}$ innen im genannten Zeitraum aufgerufen und systematisch auf $\mathrm{zu}$ extrahierende Informationen überprüft. Extrahiert wurde der $\mathrm{Na}$ me der Institution/Organisation, die Webadresse, das Datum der Erhebung sowie Inhalte mit Bezug zur Fragestellung. Die inhaltsanalytische Zuordnung bzw. Ratings in den Antwortkategorien wurden in einem separaten Schritt durchgeführt. Abweichungen zwischen den Autor ${ }^{\star}$ innen traten in etwa $10 \%$ der besuchten Internetpräsenzen auf. Diese wurden gemeinsam diskutiert und eine Lösung konsentiert.

\section{Ergebnisse}

In $\bullet$ Tab. 1 haben wir die Ergebnisse dieser Erhebung zusammengestellt. Gelistet sind für die einbezogenen Akteure jeweils die Anzahl der geprüften Internetpräsenzen und die Häufigkeit, mit der das Thema „Versorgung Nicht-an-COVID19-Erkrankter“ angesprochen wird. Falls wir Informationen zu dem Thema gefunden haben, sind weitere Charakteristika der Informationen summarisch und im Überblick zusammengestellt (z. B. Adressat ${ }^{*}$ innen, Inhalte). Ergänzende Materialien zu • Tab. 1 können unter der Korrespondenzadresse erfragt werden.

\section{Medizinische Fachgesellschaften und Kassen(zahn)ärztliche (Bundes-)Vereinigungen}

Im Erhebungszeitraum 23.04.2020-16. 05.2020 wurde die Versorgung von Menschen mit nichtübertragbaren Erkrankungen von 23 der 179 medizinischen Fachgesellschaften, 8 der 18 kassenärztlichen (Bundes-)Vereinigungen (KVen) und 8 der 18 kassenzahnärztlichen (Bundes-)Vereinigungen (KZVen) thematisiert. Dabei richteten sich die Fachgesellschaften und die KZVen jeweils etwa paritätisch an die Fachöffentlichkeit und an die Allgemeinbevölkerung bzw. Patient*innen.

\section{Organisationale Gesundheitskom- petenz auf der Mesoebene}

Auf der Mesoebene appellierten 3 Fachgesellschaften ausdrücklich an die Fachöf- fentlichkeit in den Einrichtungen der Gesundheitsversorgung, die Versorgung aufrechtzuerhalten (Radiologie, Kinderund Jugendmedizin, Urologie); weitere 3 Fachgesellschaften empfehlen, nicht dringliche Behandlungen $\mathrm{zu}$ verschieben (Gynäkologie, Chirurgie). Ausführliche Empfehlungen für das Infektionsschutzmanagement in der Pandemie wurden von 7 der 179 medizinischen Fachgesellschaften veröffentlicht (Allgemeinmedizin, Chirurgie, Gynäkologie, Krankenhaushygiene). Empfohlene Maßnahmen waren u. a. Aufteilung in getrennte Behandlungseinheiten (COVID19/Nicht-COVID-19), um Infektionen und die Sorge von Patient*innen vor einer Infektion zu reduzieren [42], Befristung von Maßnahmen, die Zugang und Inanspruchnahme gesundheitlicher Versorgung durch nicht an COVID19 erkrankte Menschen einschränken [43], verbessertes Terminmanagement für kurze Wartezeiten in Praxisräumen [44], Umstellung auf Telefon- bzw. Videosprechstunden und digitale Rezepte, um Konsultationen ohne Infektionsrisiko zu gewährleisten und gemeinsam mit Patient ${ }^{*}$ innen gute Entscheidungen über die Inanspruchnahme von Gesundheitsversorgung in der Pandemie zu treffen [45].

Informationen für Mitglieder stellten 9 KVen und 6 KZVen bereit. Inhaltlich wurden primär organisationspraktische Aspekte der ambulanten Gesundheitsversorgung in der Pandemie dargestellt, wie z.B. Schutzkleidung, arbeitsrechtliche Bestimmungen, Testungen und Meldepflicht. Informationen zur Videosprechstunde wurden von knapp einem Drittel der KVen (5 von 18) und einer der KZVen (1 von 18) bereitgestellt. Bei 4 der KZVen werden Kurzarbeit und Liquiditätshilfen bei Praxisschließungen thematisiert.

\section{Organisationale Gesundheitskom- petenz auf der Mikroebene}

Von den medizinischen Fachgesellschaften wurden bis Mitte Mai 2020 insgesamt 12 Pressemitteilungen herausgegeben; 11 davon appellierten an Menschen mit (bestimmten) nichtübertragbaren Erkrankungen, in der COVID-19-Pandemie nicht auf Akutversorgung oder
Impfungen $\mathrm{zu}$ verzichten bzw. medikamentöse Behandlungen aufrechtzuerhalten (Kinder- und Jugendmedizin, Kardiologie, Nephrologie, Orthopädie, Rheumatologie, Onkologie, Schmerz). Je nach Fachgebiet werden entsprechende Empfehlungen für spezifische Infektionsschutzmaßnahmen gegeben und/ oder auf Unterstützungsangebote hingewiesen (z. B. auf eine Schmerzhotline [46]). In 4 der Pressemitteilungen werden Patient ${ }^{*}$ innen gebeten, vorübergehend auf bestimmte Versorgungseinrichtungen auszuweichen (z. B. ambulante Praxis für Unfallchirurgie statt Klinikambulanz [47]).

Die Kassenzahnärztliche Bundesvereinigung (KZBV) und 7 der länderspezifischen KZVen informieren ausführlich über die Infektionsrisiken sowie die Akutversorgung bei Praxisschließungen. Anfang Mai 2020 werben mehrere Kampagnen darum, die Versorgung wieder in Anspruch zu nehmen. Die KVen informieren nicht explizit über die Versorgung von nichtübertragbaren Erkrankungen.

\section{Nichtkommerzielle Anbieter von Gesundheitsinformationen}

Alle im Erhebungszeitraum 23.04.202016.05.2020 einbezogenen Gesundheitsportale von nichtkommerziellen Anbietern informieren auf Mikroebene allgemein zu SARS-CoV-2 und COVID-19, die UPD verweist dabei hauptsächlich auf andere Anbieter. 2 Portale geben Hinweise für Menschen mit spezifischen Erkrankungen. Der Krebsinformationsdienst (KID) bietet ausführliche Antworten auf Fragen, die in der Pandemie gestellt wurden. Die Empfehlungen basieren auf entsprechenden Veröffentlichungen der Fachgesellschaften, es werden konkrete Alternativen benannt, der individuelle Abwägungsprozess betont und Beratung angeboten. Das IQWIG listet in der Rubrik „Unterstützung in der Corona-Krise" einen Beitrag zu Infarktsymptomen.

Die Portale des BMG und der BZgA stellen keine eigenen Informationen zur Versorgung nichtübertragbarer Erkrankungen in der Pandemie bereit.

Von den 5 Portalen bieten der KID und die UPD individuelle persönliche 
Beratung, vornehmlich über Telefon und E-Mail an.

\section{Gesetzliche Krankenkassen}

Von den im zweiten Erhebungszeitraum (05.08.2020-01.09.2020) in die Untersuchung einbezogenen gesetzlichen Krankenkassen $(n=21)$ ist auf allen Internetpräsenzen ein telefonisches Beratungsangebot rund um COVID-19 aufgeführt. Nur 1 der Krankenkassen wendet sich explizit an Personen mit Gesundheitsbeschwerden ohne (Verdacht auf) eine COVID-19 und stellt eine Entscheidungshilfe für oder gegen die Inanspruchnahme von Versorgung zur Verfügung.

\section{Krankenhäuser und Rehabilitati- onseinrichtungen}

Aufder Internetpräsenz von 22 der 25 stationären Versorgungseinrichtungen in Freiburg, Magdeburg und Oldenburg sind auf der Mikroebene Informationen $\mathrm{zu}$ organisatorischen Aspekten wie Besuchsregelungen, Hygienemaßnahmen und Mund- und Nasenschutzpflicht veröffentlicht. Für 6 Einrichtungen ist eine Beratungshotline geschaltet, 5 Einrichtungen verweisen auf die Hotline des zuständigen Gesundheitsamtes.

\section{Diskussion}

Probleme wie ein eingeschränkter $\mathrm{Zu}$ gang zur Versorgung und nosokomiale Infektionen bestanden auch im deutschen Gesundheitssystem schon vor der Pandemie. Allerdings hat die pandemische Situation die Lage verschärft, breite Bevölkerungsschichten auf Probleme aufmerksam gemacht und individuelle patient*innenseitige Abwägungsprozesse der o.g. skizzierten Gestalt notwendiger gemacht. Nicht-an-COVID-19-Erkrankte mussten sich mit einem Problem befassen, zu dessen Verständnis und Lösung sie neues Wissen und ggf. auch neue Fertigkeiten (sprich: Gesundheitskompetenz) erwerben mussten, um $\mathrm{zu}$ einer fundierten Entscheidung zu kommen.

Wurden Patient*innen bei der Entscheidung für oder gegen die Inanspruch- nahme gesundheitlicher Leistungen aktiv, beispielsweise durch individuelle Beratungsangebote unterstützt? Und wurden Einrichtungen der Gesundheitsversorgung dabei unterstützt, Patient*innen gesundheitskompetente Entscheidungen zu ermöglichen?

Die insgesamt in die Untersuchung einbezogenen Internetpräsenzen, die die Mikroebene im Fokus haben, adressieren in erster Linie Aspekte der COVID19-Infektion selbst (z. B. Infektionswege, Symptome, Schutzmaßnahmen) und nur in Ausnahmefällen liefern sie Antworten auf die Frage, wie Menschen mit akuter oder chronischer Erkrankung, die nicht an COVID-19 erkrankt sind, zu einer informierten, gut begründeten Entscheidung über die Inanspruchnahme gesundheitlicher Versorgung kommen können. Die wenigen identifizierten Ansätze sind erkrankungsspezifisch und damit nur für bestimmte Zielgruppen geeignet.

Für die meisten Patient*innen gab es damit keine gezielten Informationen zu dem Thema. Die Fortdauer der COVID-19-Pandemie und die Möglichkeit, dass es erneut zur Einschränkung der Versorgung von Nicht-an-COVID19-Erkrankten kommt, sollten Anlass für die einbezogenen Anbieter von Gesundheitsinformationen sein, Entscheidungsunterstützungen und Beratungsangebote $\mathrm{zu}$ entwickeln, um Menschen in ihren Entscheidungen zur Inanspruchnahme gesundheitlicher Versorgung zu unterstützen. Diese könnten auch über die Pandemie hinaus einen Beitrag zu einer angemesseneren Inanspruchnahme leisten [48].

Angesichts der Bedeutung organisationaler Maßnahmen im Management der Pandemie und des Ansteckungsrisikos in Einrichtungen der gesundheitlichen Versorgung überrascht es, dass diese Aspekte - zumindest in den hier näher betrachteten Informationsangeboten - nur äußerst selten in einer für Patient*innen nachvollziehbaren und verständlichen Art und Weise beschrieben werden. Informationen und Empfehlungen, die sich an Professionen in Einrichtungen der Gesundheitsversorgung richteten (z. B. Stellungnahmen von Fachgesellschaften), beinhalteten Maßnahmen des Infektionsschutzes, die auf Mesoebene ergriffen werden sollten, um das Risiko sich mit SARS-CoV-2 zu infizieren, zu minimieren. Diese Maßnahmen können indirekt dazu beitragen, Patient ${ }^{*}$ innen in ihrer Entscheidung zu unterstützen. Größtenteils vermisst werden jedoch Appelle an Einrichtungen, diese Maßnahmen explizit an die Öffentlichkeit $\mathrm{zu}$ kommunizieren, und Beratungsangebote der Einrichtungen für Patient*innen mit Unsicherheiten.

Über unsere Ergebnisse hinausgehend ist $\mathrm{zu}$ berücksichtigen, dass unklar ist, wen die hier einbezogenen Institutionen mit ihren internetbasierten Informationen und Beratungsmöglichkeiten konkret erreichen. Die aus früheren Untersuchungen bekannte geringe Reichweite solcher Informationsangebote [49] erschwerte vermutlich die Nutzung durch breite Anteile der Bevölkerung. Bedürfnisse und Voraussetzungen für einen Einsatz von digitalen Informationsangeboten und Gesundheitstechnologie [50, 51] variieren nach der Ausprägung der individuellen Gesundheitskompetenz und soziodemografischen Merkmale. Der sozioökonomische Gradient in der Gesundheitskompetenz [23, 24, 52] ist ebenfalls bekannt. Beides kann dazu beitragen, dass Menschen Entscheidungen über die (Nicht-)Inanspruchnahme gesundheitlicher Versorgung nicht sachgerecht treffen und die Folgen von Fehlentscheidungen soziale und gesundheitliche Ungleichheiten sogar verstärken [53]. Deshalb kommt Maßnahmen auf Ebene von Institutionen und Organisationen des Gesundheitswesens, die an strukturellen und prozessualen Merkmalen der Versorgung ansetzen und damit unabhängig von der individuellen Gesundheitskompetenz die Risiken einer Infektion mit SARS-CoV-2 minimieren, eine so große Bedeutung zu.

Die vorliegende Arbeit weist eine Reihe von Limitationen auf. Wir wissen nicht, zu welchem Grad die von den Fachgesellschaften empfohlenen Maßnahmen in den Versorgungseinrichtungen tatsächlich umgesetzt worden sind und wie umfassend die Einrichtungen darüber in die Öffentlichkeit kommuniziert haben. Die Anzahl der zu dieser Frage von uns näher betrachteten stationären Behandlungseinrichtungen ist zu gering, 
um hier verallgemeinerbare Aussagen $\mathrm{zu}$ treffen. Mit unserer Fokussierung auf nichtkommerzielle Informationsangebote mit einem gesellschaftlichen Auftrag haben wir viele andere Informationsquellen, die über SARS-CoV-2 mit höherer Reichweite berichten (z.B. Internetpräsenzen der öffentlich-rechtlichen Rundfunkanstalten, Presse, kommerzielle Informationsanbieter, soziale Medien) nicht berücksichtigt. Unsere Analyse spiegelt deshalb nicht die gesamte Breite der Informationen zum Thema wider, die der Bevölkerung zur Verfügung stehen. Für die Frage, inwieweit das Gesundheitsversorgungssystem seiner Verantwortung für die Bereitstellung verständlicher und verlässlicher Information nachgekommen ist, ist diese Einschränkung allerdings von geringerer Bedeutung.

\section{Fazit}

Die Bestrebungen zur Eindämmung des neuartigen SARS-CoV-2 führen zu einem eingeschränkten Zugang und einer reduzierten Inanspruchnahme von Gesundheitsversorgung für nicht an COVID-19 erkrankte Menschen. In der derzeitigen COVID-19-Pandemie und in zukünftigen Krisensituationen sollten sowohl die Gesundheitspolitik als auch Gesundheitseinrichtungen die Bedingungen für Versorgung so gestalten, dass sie gesundheitskompetente Entscheidungen für oder gegen die Inanspruchnahme gesundheitlicher Versorgung von nichtinfizierten Personen ermöglichen. In einem erfolgreichen bevölkerungsbezogenen Pandemiemanagement manifestiert sich oGK auf der Makro-, Meso- und Mikroebene.

Das im Moment noch sehr geringe Informationsangebot sollte dringend ausgebaut und verbreitet werden. Auch angesichts der Fortdauer der COVID19-Pandemie sollten Politik und Selbstverwaltung daher jetzt ihre Bemühungen intensivieren, vorhandene vertrauenswürdige, qualitativ hochwertige unabhängige Informations- und Beratungskapazitäten auszubauen, zu stärken und deren Bekanntheitsgrad zu erhöhen. Hier ist die Rolle des am 01.09.2020 online geschalteten nationalen Gesund- heitsportals (https://gesund.bund.de/; [54]) zu diskutieren, das bestehende Informationsangebote bündelt und sich explizit an Menschen mit geringer Gesundheitskompetenz richtet.

\section{Korrespondenzadresse}

\section{Prof. Dr. med. habil. Eva Maria Bitzer}

Institut für Alltagskultur und Bewegung und Gesundheit, Fachrichtung: Public Health \& Health Education, Pädagogische Hochschule Freiburg

Kunzenweg 21, 79117 Freiburg, Deutschland evamaria.bitzer@ph-freiburg.de

Danksagung. Wir danken Jana Tempes, Melanie Messer und Sarah Uthoff, die an dem Hintergrundpapier aus dem Kompetenznetz Public Health COVID19 [41] beteiligt gewesen sind.

Funding. Open Access funding enabled and organized by Projekt DEAL.

\section{Einhaltung ethischer Richtlinien}

Interessenkonflikt. E.M. Bitzer ist stellvertretende Vorsitzende des Deutschen Netzwerks Gesundheitskompetenz und leitet zusammen mit Christian Apfelbacher die AG Gesundheitskompetenz in Kompetenznetz Public Health für COVID-19. L. Ansmann, M. Hörold, L. Lyssenko und C. Apfelbacher geben an, dass kein Interessenkonflikt besteht.

Für diesen Beitrag wurden von den Autoren keine Studien an Menschen oder Tieren durchgeführt.

Open Access. Dieser Artikel wird unter der Creative Commons Namensnennung 4.0 International Lizenz veröffentlicht, welche die Nutzung, Vervielfältigung, Bearbeitung, Verbreitung und Wiedergabe in jeglichem Medium und Format erlaubt, sofern Sie den/die ursprünglichen Autor(en) und die Quelle ordnungsgemäß nennen, einen Link zur Creative Commons Lizenz beifügen und angeben, ob Änderungen vorgenommen wurden.

Die in diesem Artikel enthaltenen Bilder und sonstiges Drittmaterial unterliegen ebenfalls der genannten Creative Commons Lizenz, sofern sich aus der Abbildungslegende nichts anderes ergibt. Sofern das betreffende Material nicht unter der genannten Creative Commons Lizenz steht und die betreffende Handlung nicht nach gesetzlichen Vorschriften erlaubt ist, ist für die oben aufgeführten Weiterverwendungen des Materials die Einwilligung des jeweiligen Rechteinhabers einzuholen.

Weitere Details zur Lizenz entnehmen Sie bitte der Lizenzinformation auf http://creativecommons.org/ licenses/by/4.0/deed.de.

\section{Literatur}

1. Tam C-CF, Cheung K-S, Lam Setal (2020) Impact of coronavirus disease 2019 (COVID-19) outbreak on outcome of myocardial infarction in Hong Kong, China. Catheter Cardiovasc Interv. https://doi.org/ $10.1002 / c c d .28943$

2. Thaler M, Khosravi I, Hirschmann MT et al (2020) Disruption of joint arthroplasty services in Europe during the COVID-19 pandemic: an online survey within the European Hip Society (EHS) and the European Knee Associates (EKA). Knee Surg Sports Traumatol Arthrosc. https://doi.org/10. 1007/s00167-020-06033-1

3. COVIDSurg Collaborative (2020) Elective surgery cancellations due to the COVID-19 pandemic: global predictive modelling to inform surgical recovery plans. Br J Surg. https://doi.org/10.1002/ bjs. 11746

4. Bayles BR, George MF, Hannah H et al (2020) Impact of the first COVID-19 shelter-in-place order in the United States on emergency department utilization, Marin County, California. medRxiv. https://doi.org/10.1101/2020.07.01.20144691

5. Lai A, Pasea L, Banerjee A et al (2020) Estimating excess mortality in people with cancer and multimorbidity in the COVID-19 emergency. medRxiv. https://doi.org/10.1101/2020.05.27. 20083287

6. Søreide K, Hallet J, Matthews JB et al (2020) Immediate and long-term impact of the COVID-19 pandemic on delivery of surgical services. Br JSurg. https://doi.org/10.1002/bjs.11670

7. Nelson B (2020) Covid-19 is shattering US cancer care. BMJ 369:m1544. https://doi.org/10.1136/ bmj.m1544

8. Fasano A, Antonini A, Katzenschlager Ret al (2020) Management of advanced therapies in Parkinson's disease patients in times of humanitarian crisis: the COVID-19 experience. Mov Disord Clin Pract 7:361-372. https://doi.org/10.1002/mdc3.12965

9. Brunner M, Stinner B, Benz SR, Grützmann R (2020) COVID-19-Pandemie: Folgen für die onkologische kolorektale Chirurgie. https://www.aerzteblatt. de/nachrichten/sw/COVID-19?s $=\& p=1 \& n=1 \&$ nid=112454. Zugegriffen:20. Mai 2020

10. Deutsches Ärzteblatt (2020) COVID-19: Verschiebung elektiver Operationen muss individuell entschieden werde. https://www.aerzteblatt. de/nachrichten/sw/COVID-19?s $=\& p=1 \& n=1 \&$ nid $=111309$. Zugegriffen: 20 . Mai 2020

11. Wissenschaftliches Institut der AOK (2020) Starker Rückgang der Krankenhaus-Fallzahlen durch Coronavirus-Lockdown bei planbaren Eingriffen, aber auch bei Notfällen. https://www.aok-bv.de/ presse/pressemitteilungen/2020/index_23739. html. Zugegriffen: 7. Juli 2020

12. Deutsches Ärzteblatt (2020) Ärzte appellieren, Arztbesuche nicht aufzuschieben. https://www. aerzteblatt.de/nachrichten/sw/COVID-19?s=\& $p=1 \& n=1$ \&nid=112413. Zugegriffen: 20 . Mai 2020

13. Deutsche Akademie für Kinder- und Jugendmedizin (2020) Zeitgerechte Gabe von Standardimpfungen gem. STIKO-Empfehlungen auch in der Pandemie mit dem neuen Coronavirus (SARS-CoV-2) wichtig. https:// www.dakj.de/pressemitteilungen/zeitgerechtegabe-von-standardimpfungen-gem-stikoempfehlungen-auch-in-der-pandemie-mitdem-neuen-coronavirus-sars-cov-2-wichtig/. Zugegriffen: 7.Sept. 2020

14. Scheidt-Nave C, Barnes B, Beyer A-K et al (2020) Versorgung von chronisch Kranken in Deutschland - Herausforderungen in Zeiten der COVID-19Pandemie. J Health Monit 5:1-28. https://doi.org/ $10.25646 / 7167$

15. Büntzel J, Klein M, Keinki C, Walter S, Büntzel J, Hübner J (2020) Oncology services in corona times: 
a flash interview among German cancer patients and their physicians. J Cancer Res Clin Oncol. https://doi.org/10.1007/s00432-020-03249-z

16. Lazzerini M, Barbi E, Apicella A, Marchetti F, Cardinale F, Trobia G (2020) Delayed access or provision of care in Italy resulting from fear of COVID-19. Lancet Child Adolesc Health 4:e10-e11. https://doi.org/10.1016/S2352-4642(20)30108-5

17. Hübner J (2020) Regelversorgung: Angst vor der Isolation. Dtsch Arztebl 117(25):A-1266/B-1069

18. Chang H-J, Huang N, Lee C-H, Hsu Y-J, Hsieh C-J, Chou Y-J (2004) The impact of the SARS epidemic on the utilization of medical services: SARS and the fear of SARS. Am J Public Health 94:562-564. https://doi.org/10.2105/ajph.94.4.562

19. Zhou Q, Gao Y, Wang X et al (2020) Nosocomial infections among patients with COVID-19, SARS and MERS: a rapid review and meta-analysis. Ann Transl Med 8:629. https://doi.org/10.21037/atm20-3324

20. Carter B, Collins JT, Barlow-Pay F et al (2020) Nosocomial COVID-19 infection: examining the risk of mortality. The COPE-nosocomial study (COVID in Older PEople). J Hosp Infect. https://doi. org/10.1016/j.jhin.2020.07.013

21. Moynihan R, Johansson M, Maybee A, Lang $E$, LégaréF (2020) Covid-19: an opportunity to reduce unnecessary healthcare. BMJ 370:m2752. https:// doi.org/10.1136/bmj.m2752

22. Sørensen K, van den Broucke S, Fullam J et al (2012) Health literacy and public health: a systematic review and integration of definitions and models. BMC Public Health 12:80. https://doi.org/10.1186/ 1471-2458-12-80

23. Paakkari L, Okan O (2020) COVID-19: health literacy is an underestimated problem. Lancet Public Health 5:e249-e250. https://doi.org/10.1016/ S2468-2667(20)30086-4

24. Abel T, McQueen D (2020) Critical health literacy and the COVID-19 crisis. Health Promot Int 35(6):1612-1613. https://doi.org/10.1093/ heapro/daaa040

25. Murphy LA, Harrington P, Taylor SJ et al (2017) Clinical-effectiveness of self-management interventions in chronic obstructive pulmonary disease: an overview of reviews. Chron Respir Dis 14:276-288. https://doi.org/10.1177/ 1479972316687208

26. Palumbo R (2017) Examining the impacts of health literacy on healthcare costs. An evidence synthesis. Health Serv Manage Res 30:197-212. https://doi. org/10.1177/0951484817733366

27. Papadakos JK, Hasan SM, Barnsley J et al (2018) Health literacy and cancer self-management behaviors: a scoping review. Cancer 124:4202-4210. https://doi.org/10.1002/cncr.31733

28. Taylor DM, Fraser SDS, Bradley JA et al (2017) A systematic review of the prevalence and associations of limited health literacy in CKD. Clin J Am Soc Nephrol 12:1070-1084. https://doi.org/ 10.2215/CJN.12921216

29. Schaefer C, Bitzer EM, Dierks M-L (2019) Mehr Organisationale Gesundheitskompetenz in die Gesundheitsversorgung bringen! Ein Positionspapier des Deutschen Netzwerks Gesundheitskompetenz e. V. DNGK. https://dngk. de/gesundheitskompetenz/organisationalegesundheitskompetenz-positionspapier-2019/. Zugegriffen: 1.Sept. 2020

30. Brach C, Keller D, Hernandez LM, Baur C, Parker R, Dreyer B, Schyve P, Lemerise AJ, Schillinger D (2012) Ten attributes of health literate health care organizations. NAM Perspect. https://doi.org/10. 31478/201206a
31. The Scottish Government (2017) Making it easier: a health literacy action plan 2017-2025. NHS Scotland, Edinburgh

32. Mirzoev T, Kane S (2017) What is health systems responsiveness? Review of existing knowledge and proposed conceptual framework. BMJ Glob Health 2:e486. https://doi.org/10.1136/bmjgh2017-000486

33. Ose D (2011) Patientenorientierung im Krankenhaus. Welchen Beitrag kann ein PatientenInformations-Zentrum leisten? 1. Aufl. VS, Wiesbaden (Zugl.: Bielefeld, Univ., Diss., 2011)

34. Farmanova E, Bonneville L, Bouchard L (2018) Organizational health literacy: review of theories, frameworks, guides, and implementation issues. Inquiry 55:46958018757848. https://doi.org/10. $1177 / 0046958018757848$

35. Bitzer EM, Spörhase U (2016) Was macht Menschen gesundheitskompetent? Kompetenzerwerb aus pädagogischer und Public-Health Perspektive. In: Bundeszentrale für gesundheitliche Aufklärung (Hrsg) Health Literacy/Gesundheitskompetenz. Wissenschaftliche Definitionen - empirische Befunde und gesellschaftlicher Nutzen, Bd. 20. BZgA, Köln, S21-39

36. Abdelrahman T, Ansell J, Brown C et al (2020) Systematic review of recommended operating room practice during the COVID-19 pandemic. BJS Open.https://doi.org/10.1002/bjs5.50304

37. Moralejo D, El Dib R, Prata RA, Barretti P, Corrêa I (2018) Improving adherence to standard precautions for the control of health careassociated infections. Cochrane Database Syst Rev 2:CD10768. https://doi.org/10.1002/14651858. CD010768.pub2

38. Cochrane Library (2020) Coronavirus (COVID-19): remote care through telehealth

39. Sørensen K (2018) Shifting the health literacy mindset to enhance people-centred health services. https://health.gov/news/blog/2018/ 07/shifting-health-literacy-mindset-enhancepeople-centered-health-services. Zugegriffen: 7 . Juli 2020

40. Deutsche Netzwerk Evidenzbasierte Medizin (2016) Gute Praxis Gesundheitsinformation. Z Evid FortbildQual Gesundhwes 110-111:85-92.https:// doi.org/10.1016/j.zefq.2015.11.005

41. Ansmann L, Hörold M, Tempes J et al (2020) Wie gelingen gute Entscheidungen zur Inanspruchnahme von Gesundheitsversorgung für Nicht-Covid-19-Erkrankte? https://www.publichealth-covid19.de/images/2020/Ergebnisse/ Hintergrund-HL-Zugang-V1.pdf. Zugegriffen: 1. Sept. 2020

42. Robert Koch-Institut (2020) Ergänzung zum Nationalen Pandemieplan - COVID-19-neuartige Coronaviruserkrankung. Vorbereitungen auf Maßnahmen in Deutschland. Version 1.0. https:// www.rki.de/DE/Content/InfAZ/N/Neuartiges Coronavirus/Ergaenzung_Pandemieplan_Covid. pdf?_blob=publicationFile.Zugegriffen:31. Aug. 2020

43. Deutsche Gesellschaft für Chirurgie, Deutsche Gesellschaft für Anästhesiologie und Intensivmedizin, Berufsverband der Deutschen Chirurgen, Berufsverband Deutscher Anästhesisten (2020) Gemeinsames Statement von DGCH, DGAI, BDC und BDA zur Wiederaufnahme von elektiven Operationen in deutschen Krankenhäusern. https:// dgou.de/fileadmin/user_upload/Dokumente/ News/News/2020/Wiederaufnahme_elektiver Eingriffe_Statement_2020_04_27.pdf.Zugegriffen: 7.Sept. 2020
44. Deutsche Akademie für Kinder- und Jugendmedizin (2020) Aufrechterhaltung von Impfungen und Früherkennungsuntersuchungen in der ak tuellen SARS-CoV-2 Pandemie. Stellungnahme der Kommission für Infektionskrankheiten und Impffragen der Deutschen Akademie für Kinder- und Jugendmedizin. https://www.dakj. de/wp-content/uploads/2020/03/dakj-2020 covid-19-frueherkennungsuntersuchungenund-impfungen.pdf.Zugegriffen: 7. Sept. 2020

45. Deutsche Gesellschaft für Innere Medizin (2020) "Stille Opfer" in der Corona-Krise verhindern. DGIM: Internistische Versorgung in Deutschland ist auch für Nicht-Corona-Patienten gesichert. https://www.dgim.de/fileadmin/user_upload/ PDF/Pressemeldungen/DGIM_PM_Versorgung Innere_Medizin_F.pdf.Zugegriffen:7.Sept. 2020

46. Deutsche Schmerzgesellschaft (2020) Zugang zur Schmerztherapie derzeit noch schwieriger als sonst. https://www.schmerzgesellschaft.de/ topnavi/news-presse/pressemeldungen/presse meldung-einzelansicht?tx_news_pi1\%5Baction $\% 5 \mathrm{D}=$ detail\&tx news pi $1 \% 5 \mathrm{~B}$ controller $\% 5 \mathrm{D}=$ News\&tx_news_pi $1 \% 5$ Bnews $\% 5 D=811 \&$ cHash =e8a8aceac6d7d60e7a53727fe3300577. Zugegriffen: 1. Sept. 2020

47. Berufsverband fürOrthopädie und Unfallchirurgie, Deutsche Gesellschaft für Orthopädie und Unfallchirurgie (2020) Corona-Krise: Aufruf der Orthopäden und Unfallchirurgen an Patienten zur Entlastung der Kliniken. https://dgou. de/presse/pressemitteilungen/detailansichtpressemitteilungen/artikel/corona-krise-aufrufder-orthopaeden-und-unfallchirurgen-anpatienten-zur-entlastung-der-kliniken/. Zugegriffen: 7. Sept. 2020

48. Sorenson C, Japinga M, CrookH, McClellan M(2020) Building a better health care system post-Covid19: steps for reducing low-value and wasteful care. NEJM Catal Innov Care Deliv. https://doi.org/10. 1056/CAT.20.0368

49. Hambrock U (2018) Die Suche nach Gesundheitsinformationen. Patientenperspektiven und Marktüberblick. Bertelsmann Stiftung, Gütersloh

50. Parker S, Prince A, Thomas L, Song H, Milosevic D, Harris MF (2018) Electronic, mobile and telehealth tools for vulnerable patients with chronic disease: asystematicreview and realist synthesis. BMJOpen 8:e19192. https://doi.org/10.1136/bmjopen2017-019192

51. Rolland-Harris E, Mangtani P, Moore KM (2012) Who uses telehealth? Setting a usage baseline for the early identification of pandemic influenza activity. Telemed J E Health 18:153-157. https:// doi.org/10.1089/tmj.2011.0110

52. Sokol R, Fisher E (2016) Peer support for the hardly reached: a systematic review. Am J Public Health 106:e1-e8. https://doi.org/10.2105/AJPH.2016. 303180

53. Wahrendorf $M$, Köchelmann A, von dem Knesebeck $O$ et al (2020) Verschärfen COVID19 Pandemie und Infektionsschutzmaßnahmen die gesundheitlichen Ungleichheiten? https://www.public-health-covid19.de/images/ 2020/Ergebnisse/Hintergrundpapier_SozUngl_ COVID19_final.pdf. Zugegriffen: 9. Juli 2020 (Eine Übersicht wissenschaftlicher Erkenntnisse zur möglichen Verschärfung gesundheitlicher Ungleichheiten durch die COVID-19 Pandemie und den Maßnahmen zum Infektionsschutz)

54. Bundesgesundheitsministerium (2020) Gesundheitsportal geht online. https:// www.bundesgesundheitsministerium.de/ presse/pressemitteilungen/2020/3-quartal/ gesundheitsportal.html.Zugegriffen: 1.Sept. 2020 